https://doi.org/10.26754/ojs_aem/aem.2017282080

Recibido: 11/09/17

Aragón en la Edad Media

28 (2017) 39-66

Aceptado: 17/10/17

e-ISSN: $2387-1377$

ISSN: 0213-2486

\title{
LA VERSIÓN LATINA DEL FUERO EXTENSO DE JACA DEL SIGLO XII (UNA PROPUESTA DE RECONSTRUCCIÓN DEL TEXTO)
}

\author{
THE LATIN VERSION OF THE EXTENSIVE FUERO DE JACA OF THE $12^{\text {TH }}$ CENTURY \\ (A PROPOSAL OF RECONSTRUCTION OF THE TEXT)
}

Vicente GARCÍA EDO

Universitat Jaume I, Castellón

Resumen: A principios del siglo XII, el derecho municipal de la ciudad de Jaca se desarrolló rápidamente, dando origen a un texto legislativo extenso escrito en latín, al que denominamos Fuero Extenso de Jaca. Actualmente solo conservamos algunas copias del siglo XIV de este documento, escritas en lengua romance. Recientemente hemos identificado dos importantes fragmentos en latín del Fuero Extenso de Jaca del siglo XII, que en total contienen ciento setenta y seis artículos y nos permiten reconstruir más del $60 \%$ del texto original, así como revisar algunos aspectos relacionados con la evolución del derecho de Jaca entre los siglos XI y XIII y su influencia en la redacción de los Fueros de Aragón de 1247

Palabras clave: Fuero de Jaca, Fuero latino breve de Jaca, Fuero latino extenso de Jaca, Fuero romance extenso de Jaca, Fueros de Aragón.
Abstract: In the early twelfth century, the municipal law of the city of Jaca developed rapidly, giving rise to an extensive legislative text written in Latin, which we call the Fuero Extenso de Jaca. At the moment we only have some translations of $14^{\text {th }}$ century of this document, written in Romance language. We have recently identified two important Latin fragments of the $12^{\text {th }}$ century Fuero Extenso de Jaca, which altogether contain one hundred and seventy-six articles and allow us to reconstruct more than $60 \%$ of the original text, as well as to review some aspects related to the evolution of the law of Jaca between the eleventh and thirteenth centuries and its influence in the drafting of the Fueros de Aragón of 1247.

Key words: Fuero de Jaca, Latin short Fuero de Jaca, Latin extensive Fuero de Jaca, Romance extensive Fuero de Jaca, Fueros de Aragón. 
El Fuero de Jaca está considerado como una de las expresiones de derecho local más antiguas y notables en el conjunto de los reinos medievales de la Península Ibérica. Su origen hay que buscarlo en el último cuarto del siglo XI, durante el reinado de Sancho Ramírez de Aragón, monarca que lo concedió en forma de privilegio a una población en auge en aquellos momentos y por tanto necesitada de bases jurídicas sólidas para un mejor desarrollo, más teniendo en cuenta que durante casi todo ese mismo periodo de tiempo Jaca ostentó la capitalidad del reino de Aragón, hasta el traslado provisional de ese honor a la ciudad de Huesca cuando en 1096 se conquistó a los musulmanes. A partir de finales de 1118, Zaragoza se convirtió en la tercera y definitiva capital del reino, tras caer en manos del rey Alfonso I de Aragón.

Sin embargo ese privilegio no era suficiente, ya que para poder atender las continuas necesidades de Jaca en materia jurídica se precisaba de una acción constante de la institución monárquica, algo que en aquellos tiempos el rey no podía atender con la celeridad requerida, por falta de una infraestructura administrativa lo bastante potente como para poder responder a ese tipo de demandas; pero tampoco se opondría a que algunas poblaciones aragonesas, el mejor ejemplo es Jaca, se dotaran de una legislación complementaria a la conseguida por la vía del privilegio, especialmente en materia judicial y siempre que no afectase a los intereses de la corona, con el fin de poder resolver de manera eficaz cualquier tipo de conflicto.

La iniciativa del concejo municipal de Jaca, en este caso, sirvió para llevar a cabo la redacción y publicación de un nuevo texto de derecho municipal, que fue creciendo conforme los juristas locales añadían nuevos artículos, bien derivados del dictado de sentencias emanadas de los tribunales de justicia de la ciudad, bien producto de la voluntad de actuación de sus representantes municipales, bien del empleo de fuentes legislativas anteriores actualmente no identificadas. Desconocemos los tiempos y el ritmo con que se llevó a cabo dicha redacción, sin duda alguna años, pero sí que hay varios testimonios indirectos que nos sugieren que ya estaba en vigor y era muy completo en las primeras décadas del siglo XII y, además, se conserva el destacable elogio que dedicó el rey Alfonso II de Aragón en 1187 al derecho municipal de Jaca en su conjunto, ${ }^{1}$ señalando su importancia y prestigio den-

1 El texto se contiene en un privilegio de confirmación de los antiguos fueros y costumbres de Jaca y concesión de algunos nuevos. El documento está fechado en noviembre de 1187 y ha sido publicado en varias ocasiones. Transcribimos el fragmento que nos interesa a partir de la edición de Ana Isabel 
tro y fuera del reino, lo que significa que en ese momento constituía un cuerpo legal muy sólido. ${ }^{2}$

Así pues, desde el inicio del proceso de formación del derecho municipal jacetano, podemos hablar de la existencia de $u^{3}$ Fuero Breve de Jaca otorgado por privilegio real, que actualmente se suele fechar en 1077 y conocemos a través de varias copias, siendo la más antigua la contenida en un pergamino del siglo XII del Archivo Municipal de Jaca,${ }^{4}$ e igualmente podemos afirmar la existencia de un Fuero Extenso de Jaca, resultado del esfuerzo continuado de generaciones de jacetanos por dotarse de un derecho que permitiera un buen desarrollo de sus instituciones jurídicas. Hasta ahora, sin embargo, no se ha identificado copia alguna del siglo XII o del XIII de este texto extenso, siendo su más antigua manifestación una versión en romance de comienzos del siglo XIV, publicada por Molho en 1964 y señalada con la letra A en su edición. ${ }^{5}$

A pesar de un origen y naturaleza tan distintos, esta diferencia no se hace de notar adecuada ni suficientemente en la mayor parte de los estudios existentes dedicados al Fuero de Jaca, al obviar el significado tan concreto de cada parte del mismo. Ni tan siquiera en los trabajos de Mauricio Molho, uno de los mayores especialistas en la materia, se muestra con claridad esa diferencia, tal como puede comprobarse en su amplio estudio sobre la difusión del fuero de Jaca publicado en 1958-59, en donde casi al final del mismo nos sorprende al afirmar: «La historia del derecho de Jaca, que desde un principio tiende a confundirse con el derecho aragonés, supone varias refundiciones del núcleo primitivo contenido en el Fuero de Sancho Ramírez». ${ }^{6}$ No es posible aceptar la

Sánchez Casabón (1995), Alfonso II, rey de Aragón, conde de Barcelona y marqués de Provenza. Documentos 1162-1196). Tomo II. Institución Fernando el Católico. (CSIC). Zaragoza, doc. 450, pp. 595-598. Fragmento en p. 596: «Scio enim, quod in Castella, in Navarra et in aliis terris solent venire Iaccam per bonas consuetudines et fueros ad discendos et ad loca sua transferendos...»

2 Jesús Lalinde (1976), Los Fueros de Aragón. Zaragoza, Librería General. Así lo afirma, al señalar que este texto extenso fue resultado del trabajo continuado de «un grupo de prácticos o expertos» que se dedicó «a juzgar los litigios o pleitos surgidos entre los habitantes» de Jaca (p. 25)

3 Matizaremos el número, porque en estas mismas páginas sugerimos la posibilidad de que fueran dos los privilegios concedidos a Jaca por el rey Sancho Ramírez.

4 Archivo Municipal de Jaca (P1-1/23). Con apariencia de original, se trata de una copia solemne (pero simple, no autorizada) del texto, efectuada en fecha desconocida en el siglo XII.

5 Mauricio Molho (1964), El Fuero de Jaca. Zaragoza, CSIC, pp. 13-162. De esta versión A en romance se conservan dos manuscritos, uno en la Biblioteca Nacional de España y otro en los Archivos Nacionales de Francia. Ambos contienen, con pocas variantes, la traducción al romance (en este caso el occitano) de la última versión latina del Fuero Extenso de Jaca, tal como era a comienzos del siglo XIV.

6 Mauricio Molho (1959-60) «La difusión del Fuero de Jaca». Boletín de la Real Academia de Buenas Letras de Barcelona, 28: 21-88. La cita en p. 86. 
existencia de tales refundiciones, porque a pesar de que encontremos algunos contenidos del privilegio real en el fuero de origen municipal, ambos textos continuaron siendo siempre cosas distintas, como bien se demuestra al comprobar que el privilegio de Sancho Ramírez se copió en la segunda mitad del siglo XIII en el Libro de la Cadena de Jaca, y no así el texto municipal extenso, al que se dio traslado en libro aparte, por sus especiales características.

Por lo que a su respectiva lengua de redacción se refiere, nadie pone en duda que el privilegio de Sancho Ramírez se escribió en latín, como era propio en época tan temprana, y lo mismo debería suponerse del texto extenso que se confeccionó pocos años después; pero el hecho de que todas las copias conocidas de este último estén escritas en romance, ha llevado a bastantes autores a pensar que así se redactó originalmente, cuando la realidad es que no existe en ningún reino peninsular texto legislativo extenso alguno anterior a mediados del siglo XIII escrito en lengua distinta a la latina, porque esa era la única utilizada por los juristas a la hora de redactar cualquier documento.

$\mathrm{Si}$ alguien pretendiera cuestionar ese principio general de la documentación histórica y considerar excepción el texto romance del Fuero Extenso de Jaca, las dudas también se desvanecen al poner en relación con el mismo dos amplios fragmentos de unas recopilaciones de fueros de Aragón escritas en latín y publicadas hace casi un siglo por José M. ${ }^{a}$ Ramos Loscertales, de las que él mismo afirmaba que, aunque habían sido redactadas a principios del siglo XIII, mostraban el estado del derecho de su lugar de procedencia en la centuria anterior. Y de manera rotunda añadió: «...Es indudable que el origen de ambas... ha de ser atribuido al derecho montañés y que el lugar de redacción fue Jaca...».7 Sin embargo, por la razones que fuera, no advirtió que ambos fragmentos eran restos de la primera versión latina del Fuero Extenso de Jaca, comenzado a redactar tal vez a finales del siglo xi o, desde luego, ya a partir de principios del siglo XII. En nuestra opinión, la razón principal de no hacerlo se debió al hecho de que, a comienzos del siglo xx, era absolutamente imposible que Ramos Loscertales pudiera tener una visión tan completa como la que actualmente podemos formarnos de todos los manuscritos vinculados al derecho municipal de Jaca llegados a nuestros días, ni tampoco disponía de los medios técnicos necesarios para poder procesar la información con mayores y mejores posibilidades de éxito; todo lo cual no es obstáculo para atribuirle el 
mérito del primer reconocimiento de los fragmentos citados, que transcribió a partir de dos opúsculos distintos contenidos en el códice 225 de la Biblioteca Universitaria de Zaragoza, y posteriormente los estudió y dio a conocer a través de varias publicaciones.

Han sido muchos los autores que, a partir de los trabajos de este autor conocieron la existencia de estos dos fragmentos y los utilizaron, pero tampoco llegaron a plantearse de forma bastante clara la realidad de su significado, a pesar de que a fecha de hoy siguen constituyendo una oportunidad única de aproximación directa a la versión primitiva del primer texto legislativo extenso del reino de Aragón. Esta opinión es la que defendemos en las páginas que siguen, ya que por pura lógica así parece demostrarlo la casi exacta correspondencia entre esos dos fragmentos, a pesar incluso de las lagunas textuales que contienen y algunos otros pequeños obstáculos que mencionaremos en su lugar correspondiente, con los artículos uno a doscientos ochenta y siete de la versión A en romance del Fuero Extenso de Jaca antes citada. El fragmento más breve consta de cincuenta y nueve artículos, comenzando por el número uno y sigue, casi por el mismo orden, copiando una porción muy destacable de la primera parte de la obra; y el segundo consta de ciento diecisiete artículos, que dan comienzo en el ciento sesenta y uno y llegan hasta el doscientos ochenta y siete, que aparenta ser el último en esa versión primitiva latina del Fuero Extenso de Jaca de principios del siglo XII. Con la suma de ambos podemos conocer un $61,3 \%$ de su texto, porción más que suficiente para poder seguir avanzando con más seguridad en el estudio de los orígenes del derecho jacetano, por un lado, y del derecho general aragonés, por otro.

Los dos fragmentos latinos muestran un texto más escueto que el de las versiones conocidas escritas en romance, aunque con el paso del tiempo fue objeto de numerosos cambios, interpolaciones, y también se le añadieron veintiséis nuevos artículos (hasta un total de trescientos trece), que dieron como resultado una definitiva versión en latín del Fuero Extenso de Jaca, actualmente perdida, un manuscrito de la cual se usó para redactar, también en latín, gran parte de los Fueros de Aragón promulgados por las Cortes de Huesca a comienzos de $1247 .{ }^{8}$ A finales del siglo XIII o comienzos del XIV se tradu-

8 Ese uso concreto del Fuero Extenso de Jaca, para convertirlo en derecho general de Aragón a través de los Fueros de 1247, no era obstáculo alguno para que continuara siendo derecho municipal vigente en buena parte del norte del reino de Aragón y en Navarra, así como para que se llevaran a cabo en la segunda mitad del siglo XIII y XIV traducciones en lengua occitana, y también en castellano-aragonés, únicas llegadas a nuestros días y recopiladas y publicadas por Molho. 
jo del latín al romance esta última versión del Fuero Extenso de Jaca, originando la versión A ya citada. ${ }^{9}$

En las páginas que siguen efectuamos una propuesta de reconstrucción de la versión primitiva latina del Fuero Extenso de Jaca en el siglo XII, que por su utilidad se convirtió muy pronto en referente y base de los derechos municipales de algunas de las principales ciudades y villas aragonesas, como Huesca, Zaragoza, Tarazona o Borja y, con el tiempo, también en parte muy importante de los Fueros de Aragón promulgados en 1247 por el rey Jaime I, colaborando así de forma notable a sentar las bases del derecho territorial aragonés hasta el final de la época foral.

\section{El Fuero Breve de Jaca}

A pesar de la apariencia, muchos historiadores consideran que el pergamino conocido con el nombre de Fuero de Jaca y conservado en su archivo municipal, no es el manuscrito original otorgado por el rey Sancho Ramírez sino una copia simple realizada en el siglo XII a la que se le dio el mismo aspecto que a un original. El documento no lleva indicación del día y mes en que se hizo, y en cuanto al año está fechado siguiendo el sistema de datación de la Era Hispánica, que al convertirlo al sistema actual da como resultado el año 1062, lo cual no es posible pues el reinado de Ramiro I de Aragón, padre del otorgante, finalizó en 1064, ${ }^{10}$ y eso delata la comisión de un error por parte del escribano que hizo la copia en pergamino. Esa circunstancia ha generado no pocos quebraderos de cabeza para poder establecer la datación correcta y, en la actualidad, suele aceptarse el año 1077

9 También afirmó Molho que un total de doscientos sesenta y dos (de trescientos trece) artículos de la versión A en romance del Fuero Extenso de Jaca se utilizaron para redactar los Fueros de Aragón de 1247. Teniendo en cuenta la insistencia de las Cortes reunidas en Huesca por preservar el derecho tradicional aragonés, es más que probable que los fueros jacetanos utilizados y reconvertidos también en fueros aragoneses, presentasen alteraciones mínimas respecto del manuscrito original, con lo cual y a diferencia de lo que dijo Molho y también otros muchos autores, como lo que utilizaron los juristas reunidos con motivo de las Cortes de Huesca fueron fuentes documentales escritas en latín únicamente, si revisamos el contenido de los doscientos sesenta y dos fueros aragoneses de 1247 procedentes del derecho jacetano, nos encontraremos con que podemos reconstruir casi un $84 \%$ de la última versión latina del Fuero Extenso de Jaca.

10 Roberto Viruete Erdozaín (2013), La colección diplomática del reinado de Ramiro I de Aragón (10351064), Zaragoza, Institución Fernando el Católico. El documento 197 está fechado en marzo de 1064 y contiene la donación de una casa en Samitier y unas viñas en Estall, efectuada por Ramiro I a favor de Aznar de Santa Cruz. 
como la fecha más probable, tal como propuso con muy buen criterio Antonio Ubieto. ${ }^{11}$

Nuestra opinión sobre este pergamino es la misma que la mayoritaria, en cuanto a que se trata de una copia pero, en cuanto al contenido se refiere, pensamos que no es El Fuero de Jaca sino un privilegio posterior de confirmación de fueros concedidos por el rey con anterioridad a ese acto e incluidos en el cuerpo del nuevo documento, a los que se unen unos pocos artículos más redactados en 1077 con motivo de la erección de la villa de Jaca en ciudad.

Siguiendo el articulado del texto, a partir de la distribución establecida por Mauricio Molho en veinticuatro artículos, ${ }^{12}$ las cláusulas seis a veintiuno corresponderían a un primer acto de voluntad del monarca y las cláusulas uno a cinco y veintidós a veinticuatro, corresponderían a un acto posterior. ${ }^{13}$ Faltaría, por tanto, establecer los momentos exactos de ambos estados y, atendiendo a la realidad histórica de Jaca, parece razonable situar el primero hacia 1064, aunque todavía no pueda demostrarse, justo en los inicios del reinado de Sancho Ramírez, y el segundo en el momento en que tras el advenimiento de Sancho Ramírez al trono de Pamplona y el establecimiento de la sede episcopal jacetana, todo ello en 1076, la villa de Jaca pasa a convertirse en ciudad, por lo que la propuesta de Ubieto (1077) es muy razonable. En las líneas que siguen trataremos de explicar los motivos que permiten llegar a estas conclusiones.

\section{Un posible primer privilegio (o carta de población) en torno a 1064}

La existencia del documento, que no ha llegado a nuestros días, se justificaría por varios conductos:

a) En primer lugar por su mención indirecta en el texto del pergamino de 1077 del Archivo Municipal de Jaca, en donde al comienzo del mismo

11 Antonio Ubieto Arteta (1975), Jaca: documentos municipales: 971-1269, Valencia, Anúbar: 20-22.

12 Mauricio Molho, 1964: 3-5.

13 Ya detectó esta pluralidad de fuentes: Ana María Barrero (2003), «La difusión del Fuero de Jaca en el Camino de Santiago». El Fuero de Jaca. Vol. II: Estudios. Zaragoza, El Justicia de Aragón: 113-160. El siguiente párrafo en p. 125: «Nos encontramos, por tanto, ante un documento que no responde plenamente a los fueros concedidos por Sancho Ramírez con el fin de fomentar el desarrollo de un lugar de especial significado, sino ante un conjunto normativo diverso, en su mayor parte de origen privilegiado, que, sin embargo, es utilizado en un momento dado como instrumento para dar carta de naturaleza a una aspiración no tanto de orden jurídico como simbólico y político.» 
leemos: «Hec est carta auctoritatis et confirmationis quam ego Sanctio, gratia Dei Aragonensium rex et Panpilonensium, facio vobis». El hecho de que también se hable de confirmación estaría haciendo referencia a un privilegio anterior perdido.

b) En la cláusula primera del mismo documento podemos leer: «In primis condono vobis omnes malos fueros quos abuistis usque in hunc diem quod ego constitui Iakam esse civitatem». La forma verbal «abuistis» vuelve a señalar la existencia de un derecho local anterior.

c) Mediante privilegio fechado ${ }^{14}$ el día 11 de septiembre de 1134 y conservado en el Archivo Municipal de Jaca, el rey Ramiro II de Aragón confirmó los fueros concedidos por su padre Sancho Ramírez a los hombres de la ciudad, del modo siguiente: «Placuit michi libenti animo et spontanea voluntate, ut donarem et concederem vobis illos bonos fueros quos pater meus, Sancius rex, cui requies sit, misit in Iacca». Este fragmento de texto supone únicamente una confirmación genérica del derecho privilegiado jacetano obtenido con anterioridad, poco explícita pero suficiente para demostrar su existencia.

d) Pero hay un segundo privilegio del rey Ramiro II en el citado archivo, sin fecha, al que los historiadores coinciden en atribuir la misma que la del que nos acabamos de referir. Este nuevo documento es el que más importa a nuestro objeto pues el monarca, después de referirse al apoyo recibido de los hombres de Jaca en su difícil camino para lograr el trono del reino de Aragón, les dice: «Placuit mihi, libenti animo et spontanea voluntate, et propter servicium quod michi fecistis ${ }^{15}$ et cotidie facitis, dono et concedo vobis illos bonos fueros quos pater meus Sancius rex, cui requies sit, dedit antecessoribus vestris quando populavit Iaccam.» Añade a continuación: «Et sunt tales». Nos detendremos en primer lugar en las palabras «quando populavit Iaccam», que parece indicar que nos está remitiendo a un primer momento en el proceso constituyente de la población, que encajaría perfectamente hacia 1064, en el inicio del reinado de Sancho Ramírez, quien debió promulgar un primer privile-

14 Sobre la fecha de este privilegio y el siguiente, Ubieto: 1975: 22-23.

15 Eso hizo el rey Ramiro II en agradecimiento a los hombres de Jaca «quia vos primi elegistis me in regem», es decir, porque fueron los que primero lo reconocieron nuevo rey de Aragón, tras el difícil proceso de sucesión generado por la extravagante disposición de su hermano, el rey Alfonso I, quien falleció en 1134 habiendo nombrado en testamento herederas del reino a las órdenes militares del Temple, del Hospital y del Santo Sepulcro. 
gio a modo de carta de población, actualmente perdido, en el que figurarían al menos los dieciséis artículos que aparecen transcritos en su totalidad a continuación de las palabras «et sunt tales» de la ratificación de Ramiro II de 1134, pero no usando formas verbales de presente sino de pasado. Este mismo monarca completó su confirmación añadiendo otras cuatro importantes concesiones. ${ }^{16}$

\section{Un privilegio de 1077 de confirmación y ampliación de carta de población}

Se trata del citado pergamino conocido genéricamente como Fuero de Jaca y del que interesa destacar especialmente algunos fragmentos de su texto, que lo definen como continuación y ampliación del anterior, y son los siguientes:

a) Al comienzo y tras la invocación a Cristo y la Santísima Trinidad, el rey declara que es una carta «confirmationis», aspecto que ya habíamos destacado anteriormente, lo que delata la existencia de un derecho previo, el de la supuesta carta de población, para añadir a continuación que es su voluntad «constituere civitatem in mea villa que dicitur Iaka», lo cual denota que Jaca no solo es un burgo potente, sino también sede episcopal y con su nueva consideración como ciudad se convierte además en la primera ciudad y capital del reino de Aragón, aún en gran parte por conquistar.

b) En el artículo primero y en boca del rey se indica por tres veces las razones por las cuales confirma los fueros preexistentes, que no son otras sino las de: (1) convertir la villa de Jaca en ciudad, (2) confirmar todos los buenos fueros que se le pedían, y (3) conseguir con ello que la ciudad estuviera adecuadamente poblada. En cuanto a la razón segunda se refiere, hemos de entender que esos buenos fueros son tanto los que se incorporan por vez primera al articulado como los que procedían de la primitiva carta de población; y en cuanto a la palabra «populata» de la razón tercera, habremos de entender que se refiere al hecho mismo de que con

16 Este documento también se contiene en el Libro de la Cadena del Archivo de Jaca y fue confirmado sucesivamente por el conde Ramón Berenguer IV, yerno de Ramiro II, así como por los reyes Alfonso II de Aragón y Pedro II de Aragón, lo que indica su importancia y la preocupación del concejo municipal de Jaca por asegurar su vigencia. 
los buenos fueros, es decir, las buenas leyes dadas y confirmadas, Jaca tendría un régimen jurídico mejor que favorecería su buen gobierno.

c) Así pues, en este documento nos encontramos con que los artículos uno a cinco (siendo el uno el que se contiene entre las palabras: «In primis condono $[\ldots]$ sit bene populata»), y veintidós a veinticuatro (siendo el veintidós el que se contiene entre las palabras: «Et non detis $[\ldots]$ ad infançones»), según la numeración establecida por Molho, serían los nuevos fueros aprobados en este privilegio de ampliación de la carta de población; mientras que los artículos seis a veintiuno (siendo el seis el que se contiene entre las palabras: «Dono et concedo [...] pedone armato») se corresponden con la confirmación del rey Ramiro II efectuada en el año 1134 y, por tanto, son los buenos fueros que se otorgaron en la primera carta de población otorgada por el rey Sancho Ramírez al poco tiempo de ocupar el trono, en el año 1064, o poco después.

De todo lo anterior, por tanto, podemos concluir que es bastante razonable pensar que no hubo uno sino dos documentos fundacionales en Jaca durante el reinado de Sancho Garcés, muy representativos de sus primeros años de existencia. Poco tiempo después comenzaría la redacción del derecho complementario que la ciudad necesitaba, del que probablemente nunca pudieron imaginar los juristas que dieron inicio a la tarea, la gran repercusión que en el futuro tendría sobre el derecho de todo el reino de Aragón.

\section{La versión latina del Fuero Extenso de Jaca en el siglo XII}

Como decíamos, muy pronto fue necesario disponer en Jaca de un derecho municipal complementario del obtenido de Sancho Ramírez por vía de privilegio, lo que supuso que el concejo de la ciudad llevara a cabo iniciativas sobre cuyas circunstancias poco o nada podemos decir a fecha de hoy, con las que de manera continuada se dio lugar a la confección de un nuevo texto legislativo extenso, sin una ordenación temática, por otro lado innecesaria al ser fácilmente accesible para sus usuarios más directos a través de un índice, que fue creciendo y modificándose conforme con el paso del tiempo iba siendo preciso sumar nuevos artículos, mejorar contenidos de los existentes o, incluso, suprimir algunos por obsoletos.

Como todos los textos legislativos de los siglos XI y xII y primera mitad del XIII, en cualquiera de los reinos peninsulares, el Fuero Extenso de Jaca se re- 
dactó en latín y alcanzó no solo la perfección de que fueron capaces de dotarlo los juristas que a lo largo de generaciones participaron en su redacción, sino también la extensión que consideraron necesaria, y se mantuvo vigente en la ciudad, en mayor o menor medida, al menos hasta el final de la Edad Media. ${ }^{17}$ Por evolución de la primera versión del texto, que tratamos de recuperar en estas páginas, existió una última versión del mismo de contenido más desarrollado y extenso, escrita igualmente en latín, con la que se redactaron más de tres cuartas partes de los alrededor de trescientos cincuenta Fueros de Aragón promulgados por el rey Jaime I en las Cortes de Huesca de 1247 . El resto se completó con otras fuentes legislativas, aragonesas ${ }^{18}$ y navarras ${ }^{19}$ vigentes en ese momento y escritas en esa misma lengua. Durante todo el reinado de Jaime I (+1276) y también después, los aragoneses se negaron a que se realizara cambio alguno en el texto, por temor a que se produjeran errores que pudieran provocar merma en sus derechos. Eso explica que actualmente conservemos una docena de manuscritos latinos ${ }^{20}$ de los Fueros de Jaime I, copiados en los siglos XIV y XV, y que todas las ediciones impresas de los mismos realizadas entre los siglos XV y XIX los transmitan únicamente en latín. ${ }^{21}$

A finales del siglo XIII o comienzos del XIV la última versión latina del Fuero Extenso de Jaca se tradujo al romance por voluntad del concejo municipal, otorgando a dicha traducción el mismo valor legal que tenía la ori-

17 La bibliografía existente acerca de la difusión del Fuero de Jaca a partir del siglo XII, tanto por tierras aragonesas, navarras o castellanas es abundantísima, pero no nos interesa en estos momentos tratar de la cuestión, puesto que nuestro centro de atención en estas páginas es otro muy concreto.

18 Entre las aragonesas conviene llamar la atención sobre la inclusión de la práctica totalidad del articulado de los Fueros otorgados por el rey Pedro II en las Cortes celebradas en 1208.

19 También es destacable que en la redacción de los Fueros de Aragón de 1247 se incluya una veintena de artículos procedentes del Fuero de Estella, buena parte de ellos transcritos literalmente. Vicente García Edo (2015), «Concordancias entre el Fuero de Estella de 1164 y los Fueros de Aragón de 1247». Glossae: European Journal of Legal History, 12: 359-380.

20 Fueron objeto de estudio conjunto y edición por: Antonio Pérez Martín (2010), Los Fueros de Aragón de 1247. La Compilación de Huesca. Edición crítica del texto oficial latino. Zaragoza, El Justicia de Aragón.

21 Cierto es que hay también cuatro o cinco manuscritos que contienen traducciones en lengua romance de los Fueros de Aragón, pero son resultado de iniciativas particulares de juristas para uso privado, lo que no significa que no se utilizasen bastante, pero solo como meros libros de consulta. No es momento ahora para ocuparnos de la importancia mayor o menor de estos manuscritos, que fueron recopilados y transcritos por Antonio Pérez Martín en 1999: Los fueros de Aragón: la compilación de Huesca. Edición crítica de sus versiones romances, Zaragoza, El Justicia de Aragón. Baste decir ahora que no son textos oficiales, ni homogéneos en cuanto al contenido (a diferencia de los manuscritos latinos) ni, por supuesto, la versión romance es anterior a la versión latina de los Fueros de Aragón de 1247, a pesar de ser opinión de un número significativo de autores, incluso en fechas aún relativamente recientes. 
Vicente García Edo

ginal, lo que hizo que los manuscritos en romance aumentaran notablemente y que poco a poco los latinos existentes dejaran de utilizarse y se perdieran.

\section{Reconstrucción de la versión primitiva latina del Fuero Extenso de Jaca}

Cuando comparamos el contenido de los dos fragmentos del manuscrito de Zaragoza antes citados y publicados por Ramos Loscertales, con los manuscritos de Madrid y París que conservan el texto de la versión romance A del Fuero Extenso de Jaca, publicada por Molho, no hay duda alguna de que se trata de una misma cosa, salvadas las lagunas de texto de los fragmentos, la lengua empleada en uno y otro caso, y la diferencia de forma pero no de contenido en las soluciones jurídicas propuestas en ambas versiones. Con lo cual únicamente hay que confeccionar unas tablas que permitan visualizarlo, hasta que algún día podamos realizar una edición a texto completo comparativa de las dos versiones fragmentarias latinas ahora reconocidas, con la versión A romance, y con las más de tres cuartas partes de la versión latina última del Fuero Extenso de Jaca, utilizada en la redacción de los Fueros de Aragón de 1247. ${ }^{22}$ En esta ocasión, como decimos, nos limitamos a llevar a cabo el primer paso de este proceso, con la elaboración de las referidas tablas, que permiten facilitar la comparación entre los dos fragmentos latinos del siglo XII y la versión romance A de comienzos del siglo XIV..$^{23}$

22 Conviene señalar en este punto que, de todos los manuscritos latinos de los Fueros de Aragón promulgados por Jaime I, el conservado en el Archivo de la Catedral de Tortosa, que es muy poco utilizado, es el que en nuestra opinión transmite la versión más primitiva conocida actualmente de dicho texto, lo cual la hace especialmente interesante para efectuar esta labor de reconstrucción de la versión última latina del Fuero Extenso de Jaca, a pesar incluso de los errores de transcripción que cometió su copista

23 La primera columna, comenzando por la izquierda, indica la numeración general establecida por Molho en su edición de la versión romance A del siglo XIV, que es la única completa. El total de artículos es de trescientos trece, pero los dos fragmentos llegan solo al doscientos ochenta y siete, como ya se ha indicado en el texto. En la segunda columna figuran las palabras iniciales en latín de cada uno de los artículos o fueros contenidos en los fragmentos del texto en siglo XII. En la tercera columna, figura su numeración según la edición de José M. ${ }^{a}$ Ramos Loscertales. En la cuarta columna figura su posición en el folio correspondiente del manuscrito 225 de la Biblioteca Universitaria de Zaragoza, numerado con posterioridad a las ediciones de los textos realizadas por Ramos Loscertales. En la quinta columna figura el número de orden de los textos del siglo XII, tal como se establecieron en el estudio de Martínez Doral. Esta última columna únicamente figura en la tabla del fragmento 1, pero no en la siguiente por tratarse de un opúsculo distinto. 


\section{El fragmento 1}

El fragmento más breve ${ }^{24}$ se encuentra copiado en un opúsculo que ocupa los folios 36r al 42v del citado manuscrito 225 de la Biblioteca Universitaria de Zaragoza y contiene un total de ciento siete artículos, según estableció Martínez Doral, ${ }^{25}$ de los cuales únicamente cincuenta y nueve proceden de la versión primitiva latina del Fuero Extenso de Jaca (con las salvedades que se indican), y en líneas generales todos siguen el mismo orden que la versión romance de ese mismo fuero jacetano. El resto son fragmentos procedentes de textos legislativos de derecho visigodo o de derecho canónico, que no son ahora objeto de atención.

Las incidencias detectadas en la comparación entre este fragmento latino y la versión A romance del Fuero Extenso de Jaca son las siguientes:

El fragmento latino no incluye el artículo 2 de la versión A romance que comienza con las palabras: «...Quan esdeven que pleyt... ${ }^{26}$

El artículo 10 del fragmento, figura dividido en la versión A en tres artículos consecutivos, números 11,12 y 13 .

El artículo 11 del fragmento, que comienza con las palabras: «...Sepe contingit quod homo...» no figura en la versión A, pero sí en los Fueros de 1247.

El artículo 18 del fragmento, que comienza con las palabras: «...Item, si aliquis homo ad irricandam...» lleva el número 142 en la versión A.

24 José María Ramos Loscertales (1928) «Textos para el estudio del derecho aragonés en la Edad Media. 1. Recopilación de Fueros de Aragón», Anuario de Historia del Derecho Español, 5: 389-411. A primera vista el título induce al lector a pensar que se trata de un fragmento del cuerpo legal de derecho territorial aragonés, y no ayuda a cambiar de opinión el hecho de que dicho autor, en la breve introducción a la transcripción del texto dice que se trata de una «obra de un jurista de Huesca, el cual recogió en ella y a la vez que algunos preceptos forales del Alto Aragón, disposiciones de la "Lex Visigotorum", las más de ellas adaptadas a las necesidades jurídicas de la época y la tierra, y algunos elementos procedentes, a no dudarlo, de alguna colección canónica». Como el tema de su interés era únicamente la parte relativa al derecho aragonés, solo transcribió lo que según él eran preceptos forales aragoneses. En numerosas publicaciones este fragmento se identifica como recopilación $\mathrm{RB}$

25 Ramos Loscertales no se ocupó de esas fuentes de derecho canónico. Sí lo hizo, en cambio, muchos años después, Luis-Alfonso Martínez Doral (1971) «Notas sobre un texto para el estudio del derecho aragonés en la Edad Media», Revista de Historia Jerónimo Zurita, 21-22: 247-252. Este autor considera el opúsculo que nos ocupa como «una compilación de derecho foral navarro-aragonés» (p. 247, segundo párrafo), pero en cambio cita los textos forales como fueros de Aragón (pp. 250 y ss.) siguiendo el criterio de Ramos Loscertales, lo cual no es exactamente así. Martínez Doral numeró el total de artículos del opúsculo de acuerdo con los reclamos existentes al comienzo de cada uno de ellos.

26 Mauricio Molho, 1964: 24-25 
El artículo 36 del fragmento, que comienza con las palabras: «Conditor orbis cuius ordinacionis...» no figura en la versión A, pero sí en los Fueros de 1247.

Los artículos 41 y 42 del fragmento, figuran en la versión A agrupados en el artículo 42.

\begin{tabular}{|c|c|c|c|c|}
\hline Jaca A & Fragmento 1 ms. BUZ-225 & RL & Ms. & MD \\
\hline 1 & Siquis homo accusatus & 1 & $36 r$ & 20 \\
\hline 3 & $\mathrm{Si}$ aliqua mulier & 2 & $36 \mathrm{v}$ & 21 \\
\hline 4 & Homo qui de aliqua & 3 & $=$ & 22 \\
\hline 5 & Multociens accidi & 4 & $=$ & 23 \\
\hline 6 & Mulier infanzona multociens & 5 & $=$ & 24 \\
\hline 7 & Siquis occiderit aliquem & 6 & $=$ & 25 \\
\hline 8 & Cum ullus homo pignoraverit & 7 & $=$ & 26 \\
\hline 9 & Siquis fuerit fidanza & 8 & $37 \mathrm{r}$ & 27 \\
\hline 10 & Sepe contingit quod homo & 9 & $=$ & 28 \\
\hline 11 & Cum aliqua mulier infanzona ${ }^{a}$ & $10 \mathrm{a}$ & $=$ & 29 \\
\hline 12 & De femina franca est fuerum & $10 \mathrm{~b}$ & $=$ & 29 \\
\hline 13 & De femina de villis & $10 \mathrm{c}$ & $=$ & 29 \\
\hline- & Sepe contingit quod homo querit ${ }^{b}$ & 11 & $37 \mathrm{v}$ & 30 \\
\hline 14 & Homo quidam, cum haberet & 12 & $=$ & 31 \\
\hline 15 & Dicit fuerum quod si aliquis & 13 & $=$ & 32 \\
\hline 16 & Sepe contingit quod homo mitit & 14 & $=$ & 33 \\
\hline 17 & Sepe contingit quod homo trait & 15 & $=$ & 34 \\
\hline 18 & Cum aliquis infanzon debet & 16 & $=$ & 35 \\
\hline \multirow[t]{2}{*}{19} & Homo foraneus si pignoraverit & 17 & $=$ & 36 \\
\hline & [Item, si aliquis homo ad irricandam] ${ }^{c}$ & & & \\
\hline 21 & Dicit fuerum ut si creditor & 19 & $38 \mathrm{r}$ & 38 \\
\hline 22 & Homo quidam impetebat patrem & 20 & $=$ & 39 \\
\hline 23 & Multociens evenit quod homo & 21 & $=$ & 40 \\
\hline 24 & Si homo vel femina & 22 & $=$ & 41 \\
\hline 25 & Proponebat quidam adversus & 23 & $=$ & 42 \\
\hline 26 & Per fuerum de Aragon si infanzon & 24 & $38 v$ & 43 \\
\hline
\end{tabular}




\begin{tabular}{|c|c|c|c|c|}
\hline 27 & Siquis homo infanzon & 25 & $=$ & 44 \\
\hline 28 & Pastor, per fuerum, eum sua iura & 26 & $=$ & 45 \\
\hline 29 & Sepe contingit quod homo & 27 & $=$ & 46 \\
\hline 30 & Nobilis quidam misit in pignore & 28 & $=$ & 47 \\
\hline 31 & Si quando infanzon peccierit & 29 & $=$ & 48 \\
\hline 32 & Accidit quod quidam homo & 30 & $39 \mathrm{r}$ & 49 \\
\hline 33 & Homo quidam accipit & 31 & $=$ & 50 \\
\hline 34 & Siquis emerit ullam causam & 32 & $=$ & 51 \\
\hline 35 & Siquis obtulerit contra adversarium & 33 & $=$ & 52 \\
\hline 36 & Accipit sepe et multociens & 34 & $=$ & 53 \\
\hline 37 & Homo quidam petebat & 35 & $40 \mathrm{r}$ & 78 \\
\hline- & Conditor orbis cuius ordinacione ${ }^{d}$ & 36 & $=$ & 83 \\
\hline 38 & Res in dubio posita arbitrio & 37 & $40 v$ & 84 \\
\hline 39 & Si alicui homini de iusticia & 38 & $=$ & 85 \\
\hline 40 & Per fuerum de Aragon, quicumque & 39 & $=$ & 86 \\
\hline 41 & Cum aliquis posuerit in pignore & 40 & $=$ & 87 \\
\hline $42 \mathrm{a}$ & Petebat quidam a parentibus & 41 & $41 \mathrm{r}$ & 88 \\
\hline $42 b$ & Evenit multociens quod homo & 42 & $=$ & 90 \\
\hline 43 & Comendaverat quidam homo & 43 & $=$ & 91 \\
\hline 44 & Sepe et sepius contingit & 44 & $41 v$ & 92 \\
\hline 45 & Hereditates que perveniunt filii & 45 & $=$ & 93 \\
\hline 46 & Si aliquis homo inceperit & 46 & $=$ & 94 \\
\hline 47 & Item, si aliquis aperuerit & 47 & $42 \mathrm{r}$ & 95 \\
\hline 48 & Item, si aliquis homo in quolibet & 48 & $=$ & 96 \\
\hline 55 & Item, istud fuerum inter regem & 50 & $=$ & 97 \\
\hline 56 & Item, ullus infanzon non debet & 49 & $=$ & 98 \\
\hline 57 & Item, si infanzon occiderit alium & 51 & $=$ & 99 \\
\hline 58 & Item, si aliquis infanzon occiderit & 52 & $=$ & 100 \\
\hline 59 & Item, siquis percusserit aliquem & 53 & $=$ & 101 \\
\hline 60 & Item, multi homines de signal & 54 & $=$ & 102 \\
\hline 61 & Item, inter christianum et & 55 & $=$ & 103 \\
\hline
\end{tabular}


Vicente García Edo

\begin{tabular}{|c|l|c|c|c|}
\hline 94 & Item, infanzon ermunius de furto & 56 & $=$ & 104 \\
\hline 95 & Item, omnis villa in qua est claviger & 57 & $42 \mathrm{v}$ & 105 \\
\hline 96 & Item, homo mortuus non habet & 59 & $=$ & 106 \\
\hline 97 & Item, omnis homo qui habet & 58 & $=$ & 107 \\
\hline 142 & Item, si aliquis homo ad irricandam & 18 & $38 \mathrm{v}$ & 37 \\
\hline
\end{tabular}

a En el manuscrito este fuero y los dos siguientes figuran agrupados en uno solo, mientras que en la versión romance A del Fuero de Jaca se transcriben los tres separados.

$b$ No hemos localizado su equivalente en la versión A del fuero de Jaca, pero sí en los Fueros de Aragón, edición incunable, f. XVIIIIr, fuero "Quicumque homo demandat...»

c Es el número 142 de la versión romance A, razón por la cual lo hemos cambiado de lugar.

$d$ No localizado en la versión A del Fuero de Jaca, pero sí en la edición incunable de los Fueros de Aragón, ff. XVIIIIv-XXr, fuero «Conditor orbis a quo cuncta...»

A partir del artículo 48 del fragmento 1 latino, el autor del opúsculo transcribió una decena más de artículos procedentes de la fuente que le sirvió de referencia, pero ya no todos seguidos sino seleccionando únicamente aquellos que le interesaron para su trabajo. El número de orden del penúltimo, el 58, se corresponde con el artículo $97 \mathrm{del}$ manuscrito A romance, lo que significa que este anónimo copista tuvo en sus manos un manuscrito que contenía, al menos el primer tercio del texto de la versión primitiva latina del Fuero Extenso de Jaca.

\section{El fragmento 2}

El segundo fragmento forma parte de un opúsculo que ocupa los folios $3 \mathrm{r}$ a $13 \mathrm{v}$ del manuscrito 225 de la Biblioteca Universitaria de Zaragoza y consta de un total de ciento cincuenta y cinco artículos según la numeración establecida por Ramos Loscertales, que lo publicó ${ }^{27}$ por vez primera en 1925 y lo tituló como el anterior. ${ }^{28}$ En la edición crítica del mismo

27 José María Ramos Loscertales (1925), «Textos para el estudio del derecho aragonés en la Edad Media. Recopilación de Fueros de Aragón». Anuario de Historia del Derecho Español, 2: 491-523. En muchas publicaciones este opúsculo se identifica como recopilación RA.

28 El título que le dio tampoco ha ayudado a hacer del texto algo especialmente atractivo para los historiadores del derecho aragonés a lo largo de la mayor parte del siglo xx, a pesar de que en la breve nota introductoria Ramos Loscertales dijo algunas cosas importantes (pp. 491-492), como que era una «Recopilación adaptada por un jurisconsulto, posiblemente del primer tercio del siglo xiII y de Zaragoza, para ser usada en la comarca aragonesa de riberas del Ebro. Su núcleo fundamental lo constituyen disposiciones del Fuero de Jaca. La forma de redacción conservada por la Recopilación es más antigua que la del texto lemosín de aquél. Del cotejo con uno de los manuscritos resulta: que el contenido de la mayoría de los capítulos es el mismo, siendo las variantes, por lo general, 
texto, ${ }^{29}$ efectuada en 1986 por Juan José Morales Gómez y Manuel José Pedraza García, el número de artículos se situó en ciento sesenta, y lo titularon con mejor criterio como Fueros de Borja y Zaragoza, aunque realmente sea algo más que eso. Sin entrar en mayores consideraciones, señalaremos que las diferencias numéricas se justifican sobre todo por la inclusión o no de diversas adiciones de textos que figuran a pie de página en varias hojas del manuscrito.

De acuerdo con los autores de la última edición citada, este opúsculo contiene una copia, no sabemos si completa, del estado del fuero municipal extenso de Borja hacia mediados del siglo XII, resultado de la adopción por dicha villa del derecho municipal de Zaragoza también vigente en aquellos momentos, al que añadieron un pequeño número de artículos redactados por iniciativa de su concejo municipal y perfectamente identificables en el texto. El criterio seguido para tomar tal decisión de dependencia no era otro sino el de considerar, a falta de un derecho territorial o general de Aragón, que si el modelo importado era útil en la capital del reino, también podía serlo en una población de menor tamaño, como Borja, si efectuaban los añadidos personalizadores necesarios que, en conjunto, solamente ascendieron a un total de diez artículos.

Si restamos el breve articulado relativo a Borja del manuscrito que nos ocupa, se supone que el resto es Fuero de Zaragoza y, efectivamente, así lo parece a partir de la lectura de los nueve primeros artículos, que se redactaron en la capital del reino de Aragón, pero a partir del décimo el discurso cambia y nos encontramos de nuevo con la versión primitiva latina del Fuero Extenso de Jaca, pero no desde el primer artículo sino a partir del ciento setenta (según la numeración de referencia de la versión romance A) y sigue adelante, por el

interpolaciones aclaratorias del sentido, constituyendo excepción las alteraciones esenciales del texto...».

29 El texto era, sin embargo, algo más que la mera recopilación de un jurisconsulto, y así debieron estimarlo Juan José Morales y Manuel José Pedraza, dirigidos por el profesor Antonio Ubieto Arteta, a la hora de llevar a cabo en 1986 una nueva edición, precedida de un brevísimo texto introductorio, a pesar de lo cual dan algunas claves para una mejor interpretación del mismo puesto que, efectivamente, el manuscrito nos transmite una versión de los llamados Fueros de Zaragoza y Borja, a partir de una copia procedente de esta última población, puesto que hacia el final del manuscrito se transcriben algunos artículos en los que se hace mención expresa a la misma. No sucede lo mismo con aquellos que pudieran corresponder a Zaragoza. Ambos autores hacen otras atinadas observaciones, que no compartimos totalmente, que les permiten concluir que entre 1134 y 1151, debieron redactarse los fueros específicos de Borja que forman parte del manuscrito. Especialmente significativa es la referencia a un artículo en el que se señala la existencia de los monjes cluniacenses o monjes negros, establecidos en el monasterio de Veruela a partir de 1144, lo cual consideran que es un dato más para acercarnos al momento en que todo el articulado del manuscrito tendría vigencia en la población 
mismo orden aunque también con algunas incidencias que señalaremos, hasta el artículo doscientos ochenta y siete de la citada versión romance.

Sabemos que los habitantes de Tarazona adoptaron las mismos criterios que los de Borja, también en fechas muy tempranas, y aunque no ha llegado a nuestros días un manuscrito de características similares al que estamos comentando relativo a esa población, sí que se conoce el texto de un contrato de compraventa $^{30}$ fechado en 1139 , en el que se daba fianza de salvedad para garantizar su cumplimiento «ad forum de Saragoza et Tarassona», lo que viene a referirse a un manuscrito similar al que nos ocupa, en ese caso con el articulado del Fuero de Zaragoza más el propio ordenado por el concejo municipal de Tarazona.

Desconocemos cuándo comienza a formarse un fuero municipal extenso en Zaragoza, tras su conquista a los musulmanes en diciembre de 1118. Sabemos únicamente que muy pocos días después de este hecho el rey Alfonso I de Aragón concedió ${ }^{31}$ a los nuevos pobladores de la ciudad los fueros de los infanzones de Aragón, si bien se trata de un documento muy breve y escueto y, por ese motivo, insuficiente para un buen desarrollo de la nueva capital del reino de Aragón; razón por la cual sus representantes municipales debieron dirigir muy pronto la mirada hacia el derecho municipal de Jaca, que ya tenía un cierto recorrido y garantizada su eficacia, y no dudaron en adoptarlo como propio, si bien a fecha de hoy y a tenor del opúsculo al que nos estamos refiriendo únicamente podemos afirmarlo respecto de lo contenido en el mismo. Parece razonable pensar, sin embargo, que toda la versión latina vigente en esos momentos del Fuero Extenso de Jaca, sirvió de referente para construir un Fuero de Zaragoza propio, tras añadir artículos nuevos para atender singularidades de la ciudad, pero a fecha de hoy esto no pasa de ser una mera hipótesis.

Lo que sí que podemos afirmar, en cambio, es que en una fecha tan temprana como el 24 de abril de 1124 ya encontramos una primera referencia, ${ }^{32}$ tal vez pudiera haber alguna anterior, al «forum de Cesaraugusta», citado al establecer la fianza de salvedad en un contrato de compraventa de un huerto en los arrabales de Zaragoza. De 1125 es una segunda referencia, en este caso indirecta, a un documento que no hemos podido averiguar si se ha publicado en alguna parte,

30 Publicado por José María Lacarra (1982), Documentos para el estudio de la reconquista y repoblación del Valle del Ebro. Vol. 1., Zaragoza, p. 286, documento 287.

31 Lacarra, 1982: 71-72 (documento 57).

32 Lacarra, 1982: 113 (documento 99). 
mediante el cual la Orden del Temple concedió a los hombres de Novillas, ${ }^{33}$ localidad próxima a Mallén ${ }^{34}$ y Borja, una carta puebla, mediante la cual se les otorgaban diversas franquezas y además el fuero de Zaragoza como derecho supletorio del local. Interesa señalar, de ser correcta la cita, que en fecha tan temprana es la primera vez que se documenta la aplicación del derecho municipal zaragozano a otro municipio; algo que a partir de ese momento será muy frecuente pues, el Fuero de Zaragoza, se extendió a lo largo de la segunda mitad del siglo XII y la primera del siglo XIII no solo por una gran parte de la geografía aragonesa, sino también fuera de ella. Este fenómeno está perfectamente documentado en buena parte de las tierras de la actual provincia de Castellón desde el inicio mismo de las campañas militares de conquista del reino de Valencia, y se efectuó a través de la concesión de numerosas cartas de población a villas y lugares sometidos a jurisdicciones de muy distintos señores, sobre todo entre los años 1233 y $1246 .{ }^{35}$

Al estar basado el Fuero de Zaragoza en gran parte sobre la versión primitiva latina del Fuero de Jaca, éste indirectamente también se estaba aplicando en los territorios citados, de manera que con el paso del tiempo muchas de las instituciones jurídicas jacetanas se conocían y aplicaban, al menos teóricamente, en gran parte del reino aragonés con anterioridad a la promulgación de los Fueros de Aragón de 1247. Con lo cual se justifica todavía más la insistencia de los brazos de las Cortes en adoptar el derecho propio vigente en el reino, y no ceder a la voluntad del rey Jaime I, deseoso de implantar en Aragón un derecho basado en la recepción del «ius commune» procedente de Italia, una corriente de pensamiento jurídico de moda en Europa Occidental en el siglo XIII sustentada en el antiguo derecho romano-justinianeo, revitalizado desde finales del siglo XI en la ciudad de Bolonia. Si los Fueros de Ara-

33 La cita al documento se contiene en la Colección de fueros y cartas pueblas de España por la Real Academia de la Historia (1852). Madrid, p. 163. Por aquellas fechas se indica que el documento original, suponemos que sobre pergamino, se encontraba en el Archivo de la Orden de San Juan en Zaragoza, encomienda de Novillas, legajo $3, \mathrm{n}^{\circ}{ }^{\circ}$. En 1877 este archivo se trasladó a Alcalá de Henares y posteriormente pasó al Archivo Histórico Nacional, en Madrid.

34 En junio de 1132 Alfonso I de Aragón concedió privilegios y franquicias a los mozárabes de Mallén y les otorgó también el derecho de Zaragoza «...dono vobis foros, consuetudines quales habent Caesaraugusta et Tutela...» María Luisa Ledesma Rubio (1991), Cartas de población del reino de Aragón en los siglos medievales, Zaragoza, Institución Fernando el Católico, pp. 56-57 (documento 56). La mención exacta al mismo dice: «...Etiam dono vobis, foros, consuetudines, quales habent Cesaraugusta et Tutela...».

35 Antonio Ubieto Arteta (1989), «Los precedentes de los Fueros de Aragón». En Vidal Mayor. Estudios. Huesca, Diputación Provincial-Instituto de Estudios Altoaragoneses: 30-33. 
gón se hubieran redactado según criterio (de la cancillería) de Jaime I, eso hubiera supuesto romper con una tradición jurídica propia y comenzar de nuevo, algo a lo que los aragoneses no se plegaron nunca sino que consiguieron mantener el derecho propio, de amplia base jacetana, como fuente principal de su nuevo derecho territorial.

En fechas todavía recientes llevamos a cabo la publicación de un estudio sobre el Fuero de Zaragoza ${ }^{36}$ en el siglo XII y pudimos singularizar todos los fueros relativos a esta ciudad dentro del manuscrito que nos ocupa, que son un total de treinta y cuatro. Si los restamos del texto, después de restar también los de Borja, lo que nos queda son un total de ciento diecisiete artículos correspondientes a la tercera parte final de la versión latina del Fuero Extenso de Jaca en las primeras décadas del siglo XII.

Para el cómputo global, sin embargo, hay que señalar que en esta ocasión encontraremos préstamos del derecho jacetano no solo en el cuerpo del manuscrito, sino también en un par de adiciones a pie de página, efectuadas por mano de un segundo copista y como es natural con posterioridad a la redacción de este fragmento segundo, lo que significa que se copiaron en la primera mitad del siglo XIII. La primera está en el folio $3 \mathrm{v}$ y da comienzo con las palabras «Item, qui locat bestiam...», que se corresponde con el fuero ciento setenta y uno de la versión romance del Fuero de Jaca, lo que parece dar a entender que, salvo que se tratase de un olvido del primer copista, nos encontraríamos con un nuevo fuero jacetano adicionado con bastante posterioridad a la redacción de la masa principal del mismo, que se confeccionó en las primeras décadas del siglo XII. Lo mismo sucede con otra adición al pie del folio 12r, que comienza así: «De pleito christiani et mauri...», que se corresponde con el fuero doscientos setenta y seis de la versión romance del Fuero de Jaca y, por tanto, debió ser adición a la masa principal.

También convendría señalar que hay cambio de ubicación de un par de fueros entre el manuscrito latino y el romance, que hemos modificado a beneficio de este último, porque marca la pauta en cuanto a la numeración general se refiere. Así pues, el artículo cincuenta y tres del fragmento latino se corresponde con el doscientos ochenta y siete del manuscrito A romance, con lo cual en nuestra tabla lo hemos de situar al final de la misma; pero en cambio el artículo cincuenta y cuatro de este fragmento se corresponde con el artículo

36 Vicente García Edo (2016), «El Fuero de Zaragoza en el siglo XII. (Aproximación a su estudio)». Revista de Dret Històric Català, 15: 165-197. 
ciento sesenta y uno de la citada versión romance, con lo cual lo hemos de avanzar de posición. Como el cuerpo principal del manuscrito finalizó ocupando el tercio superior del folio $13 \mathrm{v}$, con el artículo «De domino et vassallo qui sunt affidançati», que ocupa la posición doscientos ochenta y seis de la versión A romance del Fuero de Jaca, parece razonable pensar que en la primera mitad del siglo XII ese era aproximadamente el punto en el que finalizaba este texto legislativo, y en las décadas posteriores se seguirían efectuando añadidos hasta alcanzar el número de trescientos trece artículos que contiene la versión romance $\mathrm{A}$.

En la tabla a continuación podemos ver el paralelismo entre la parte procedente del derecho de Jaca en este opúsculo y la versión manuscrita romance, siguiendo el mismo esquema que en el anterior fragmento, aunque adecuada a las características del manuscrito.

\begin{tabular}{|c|l|c|c|}
\hline Jaca A & Fragmento 2 ms. BUZ-225 & RL & Ms. \\
\hline 161 & De venditione aut pignore & 54 & $6 \mathrm{v}$ \\
\hline 170 & Si sunt duo equi ligati & 10 & $3 \mathrm{v}$ \\
\hline 171 & De qui locat bestiam ${ }^{a}$ & margen & $=$ \\
\hline 172 & De ferrum levare in villa & 11 & $=$ \\
\hline 173 & Qui impignorat cultellum & 12 & $=$ \\
\hline 174 & De spata impignorata, et est ibi & 13 & $=$ \\
\hline 175 & De qualibet arbore avulsa & 14 & $=$ \\
\hline 176 & Quilibet homo dabit fidantiam & 15 & $=$ \\
\hline 177 & De fidantia que intrat & 16 & $4 \mathrm{r}$ \\
\hline 178 & De pediare hereditatem. Si est & 17 & $=$ \\
\hline 179 & De placito sinato per duorum & 18 & $=$ \\
\hline 180 & De homine qui crebantat & 19 & $=$ \\
\hline 181 & De hereditate quam impig. & 20 & $=$ \\
\hline 182 & De amicis qui impignorant & 21 & $=$ \\
\hline 183 & De homine qui dat suum & 22 & $=$ \\
\hline 184 & De duobus molendinis qui fuerint & 23 & $=$ \\
\hline 185 & De pignoribus que faciunt & 24 & $4 \mathrm{v}$ \\
\hline 186 & De muro ville. Si tenet & 25 & $=$ \\
\hline 187 & De duobus hominibus qui vadunt & 26 & $=$ \\
\hline
\end{tabular}




\begin{tabular}{|c|c|c|c|}
\hline 188 & De balneo et furno et taberna & 27 & $=$ \\
\hline 189 & De hominibus qui intrant & 28 & $=$ \\
\hline 190 & De hominibus ordinum & 29 & $=$ \\
\hline 191 & De dominus ville qui habet & 30 & $=$ \\
\hline 192 & De homine qui dat saltum & 31 & $5 \mathrm{r}$ \\
\hline 193 & De homine qui comparat & 32 & $=$ \\
\hline 194 & De cabeçalariis et testibus & 33 & $=$ \\
\hline 195 & De domibus infançonis & 34 & $=$ \\
\hline 196 & De homine qui demandat & 35 & $=$ \\
\hline 197 & De casis et tentoris duorum & 36 & $=$ \\
\hline 198 & De hereditate commendata & 37 & $=$ \\
\hline 199 & De homine qui vadit per mercatum & 38 & $=$ \\
\hline 200 & De tota ferida de spata & 39 & $=$ \\
\hline 201 & De molendino qui colat de die & 42 & $5 v$ \\
\hline 202 & De homine qui dat hereditatem & 43 & $=$ \\
\hline 203 & De cane qui intrat per tectum & 44 & $=$ \\
\hline 204 & De gallinis et apibus et palomis & 46 & $=$ \\
\hline 205 & De homine qui reddit se & 47 & $6 \mathrm{r}$ \\
\hline 206 & De duobus clamantibus & 48 & $=$ \\
\hline 207 & De homine alterius terre & 49 & $=$ \\
\hline 208 & De homicidio habet accipere & 50 & $=$ \\
\hline 209 & De desfillamento patris ad filium & 55 & $6 \mathrm{v}$ \\
\hline 210 & De cane custode domus & 56 & $=$ \\
\hline 211 & De testamento infançonis & 57 & $7 \mathrm{r}$ \\
\hline 212 & De bove aut qualibet alia bestia & 59 & $=$ \\
\hline 213 & De bestia duorum participum & 60 & $=$ \\
\hline 214 & De iuramento testium. Duo testes & 61 & $=$ \\
\hline 215 & De morte canis. Si occidit & 62 & $7 \mathrm{v}$ \\
\hline 216 & De testibus qui sunt in carta & 64 & $=$ \\
\hline 217 & De pignora porcorum & 65 & $=$ \\
\hline 218 & De testibus qui exeunt per asinum & 66 & $=$ \\
\hline 219 & De pignorare. Si vis aliquem & 67 & $=$ \\
\hline 220 & De duobus amicis postquam & 68 & $=$ \\
\hline 221 & De cane venatico. Si occidit & 71 & $8 \mathrm{r}$ \\
\hline
\end{tabular}




\begin{tabular}{|c|c|c|c|}
\hline 222 & De percussione infançonis & 72 & $=$ \\
\hline 223 & De homine qui non debet facere & 73 & $=$ \\
\hline 224 & De villano percusso & 74 & $=$ \\
\hline 225 & De homine qui intrat in iudicium & 75 & $=$ \\
\hline 226 & De homine infirmo reptato & 76 & $=$ \\
\hline 227 & De milite qui facit ricum hominem & 77 & $8 v$ \\
\hline 228 & De homine que ivit in mercadariam & 78 & $=$ \\
\hline 229 & Fuit facta façania in valle de Funes & 79 & $9 \mathrm{r}$ \\
\hline 230 & Quidam homo dedit grandem & 80 & $=$ \\
\hline 231 & De homine qui vadit per viam & 81 & $=$ \\
\hline $232 \mathrm{a}$ & De iuramentis cristianus & 82 & $9 v$ \\
\hline $232 b$ & Iudeus habet iurare christiano & 83 & $=$ \\
\hline 233 & De bestia accomodata vel conducta & 84 & $=$ \\
\hline 234 & De quolibet homine qui accomodat & 85 & $=$ \\
\hline 235 & De quolibet fructu vendito & 86 & $=$ \\
\hline 236 & De testibus falsis. Si probati sunt & 87 & $=$ \\
\hline 237 & De homine periuro et probato & 88 & $=$ \\
\hline 238 & De homine qui intrat in iudicium & 89 & $=$ \\
\hline 239 & De homine qui pignorat dominum & 90 & $=$ \\
\hline 240 & De furno et molendino & 91 & $=$ \\
\hline 241 & De aqua furata & 92 & $10 \mathrm{r}$ \\
\hline 242 & De filio borde qui demandat & 93 & $=$ \\
\hline 243 & De arboribus que fuerint plantati & 94 & $=$ \\
\hline 244 & Palum de parrali & 95 & $=$ \\
\hline 245 & De comanda quarumlibet rerum & 96 & $=$ \\
\hline 246 & De christiano qui pignorat & 97 & $=$ \\
\hline 247 & De homine qui dat fidantiam & 98 & $=$ \\
\hline 248 & De fidantiis movilis aut terribilis & 99 & $=$ \\
\hline 249 & De matre et patre qui dant & 100 & $=$ \\
\hline 250 & De homine qui tenet placitum & 101 & $10 \mathrm{v}$ \\
\hline 251 & De patre aud matre qui fuerint & 102 & $=$ \\
\hline 252 & De spata vel cultello & 103 & $=$ \\
\hline 253 & De partitione germanorum & 104 & $=$ \\
\hline 254 & De homine vel femina & 105 & $=$ \\
\hline
\end{tabular}




\begin{tabular}{|c|c|c|c|}
\hline 255 & De homine qui dicit se daturum & 106 & $=$ \\
\hline 256 & De debitis clericorum & 107 & $=$ \\
\hline 257 & De homine qui monstrat cartam & 108 & $11 \mathrm{r}$ \\
\hline 258 & De filio vel filia qui miserunt & 109 & $=$ \\
\hline 259 & De homine qui vult probare & 110 & $=$ \\
\hline 260 & De testibus patris contra filium & 111 & $=$ \\
\hline 261 & De homine qui dicit & 113 & $=$ \\
\hline 262 & De homine qui est in hora mortis & 114 & $=$ \\
\hline 263 & De omnibus causis que fuerint & 115 & $=$ \\
\hline 264 & De quinque solidis illius sume & 116 & $=$ \\
\hline 265 & De homine qui se clamat & 117 & $=$ \\
\hline 266 & De homine qui pignorat et occidit & 118 & $11 \mathrm{v}$ \\
\hline 267 & De iuramento qui coactione domini & 119 & $=$ \\
\hline 268 & De fidantia que est et dicit & 120 & $=$ \\
\hline 269 & De asino vel asina & 121 & $=$ \\
\hline 270 & De hominibus qui testantur & 122 & $=$ \\
\hline 271 & Façania. Quidam iudeus obligavit & 124 & $12 \mathrm{r}$ \\
\hline 272 & De hereditate germanorum & 125 & $=$ \\
\hline 273 & De homine qui pignorat in villa & 126 & $=$ \\
\hline 274 & De gato furato et invenitur & 127 & $=$ \\
\hline 275 & De causa clerici et laici & 128 & $=$ \\
\hline 276 & De pleito christiani et mauri ${ }^{6}$ & margen & $=$ \\
\hline 277 & De homine albarrano qui non & 130 & $=$ \\
\hline 278 & De testibus postquam sunt & 132 & $12 \mathrm{v}$ \\
\hline 279 & De interfectoribus venatuum & 133 & $=$ \\
\hline 280 & De homine qui vadit post venatum & 134 & $=$ \\
\hline 281 & De venato qui cadit in cepum & 135 & $=$ \\
\hline 283 & De homine qui tenet tortum & 140 & $13 \mathrm{r}$ \\
\hline 284 & De homine qui se clamat & 142 & $=$ \\
\hline 285 & De vassallo qui sedet ad soldatam & 149 & $13 \mathrm{v}$ \\
\hline 286 & De domino et vassallo qui sunt & 150 & $=$ \\
\hline 287 & De foro quem dedit dominus rex & 52 & $6 v$ \\
\hline
\end{tabular}

a Este fuero también figura añadido a pie de página, y se corresponde con el fuero 276 de la versión A romance del Fuero de Jaca. 


\section{Conclusiones}

1. Parece razonable considerar que el origen del derecho municipal de Jaca se encuentra en un privilegio concedido por el rey Sancho Ramírez a la villa a comienzos de su reinado que no ha llegado a nuestros días, aunque sí se ha conservado la parte más sustancial de su articulado, transcrito y confirmado por el rey Ramiro II en 1135; pero no podemos descartar que algunos artículos primitivos fueran suprimidos por distintas razones al promulgar el privilegio que figura a continuación.

2. Un segundo privilegio del mismo Sancho Ramírez, otorgado en 1076-77 a Jaca con ocasión de elevarla a la categoría de ciudad, sirvió para confirmar el derecho existente en esos momentos, que se transcribió en el cuerpo del documento y al que se añadieron varios nuevos artículos. Sea uno o sean dos los privilegios que acabamos de citar, este último reúne toda la información llegada a nuestros días del primer derecho privilegiado de Jaca, por lo que en adelante deberíamos denominarlo Fuero Latino Breve de Jaca.

3. Como el articulado del Fuero Breve no era suficiente para las necesidades de la ciudad, en pleno desarrollo en el paso del siglo XI al XII, el concejo municipal de Jaca promovió la redacción, también en latín, de un derecho propio complementario del privilegiado que ya tenía, que fue creciendo rápidamente y en las primeras décadas del siglo XII contaba al menos con doscientos ochenta y siete artículos, adquiriendo buena fama por su singularidad y contenido, de manera que muy pronto superó los límites del municipio y comenzó a utilizarse también dentro y fuera de Aragón: en tierras de Navarra lo adoptaron con enorme fuerza y amplitud, pero aquí interesa destacar más su pronta extensión a las poblaciones de Zaragoza, Novillas, Mallén, Tarazona y Borja.

4. El derecho local de Jaca o, sus «bonas consuetudines», según las denominó Alfonso II de Aragón en 1187 constituyen, en la primitiva redacción latina parcialmente reconstruida en páginas anteriores, el punto de partida para replantear el origen y posterior desarrollo del derecho aragonés durante los siglos XII, XIII y comienzos del XIV, hasta la estabilización de las Cortes Aragonesas como principal institución productora de derecho para el conjunto del reino de Aragón. Sería oportuno, por tanto, denominar a partir de ahora a este texto recuperado como Fuero Latino Extenso de Jaca, con el fin de dotarlo de identidad propia y no confundirlo con la versión breve latina del mismo, ni tampoco con la versión extensa romance, antes citadas. 
5.- Las consecuencias inmediatas que se derivan tras la reconstrucción parcial de la versión primitiva del siglo xII del Fuero Latino Extenso de Jaca son, entre otras, las siguientes:

- Borrará cualquier duda acerca de cuál fue la lengua original de redacción de la versión primitiva del Fuero Extenso de Jaca, que no fue otra sino el latín.

- Demuestra que los Fueros de Aragón de 1247 se redactaron originariamente en latín, en gran parte tomando como referencia principal la versión última del Fuero Latino Extenso de Jaca, en la misma lengua que las otras fuentes documentales de dichos Fueros identificadas hasta el momento, por lo que no hubo nunca traducción del romance al latín de los fueros aragoneses, sino más bien lo contrario y con bastante posterioridad a su promulgación, es decir, cuando comenzaron a efectuarse por iniciativa privada distintas traducciones de los Fueros de Aragón, tanto en lengua occitana como en castellano-aragonés, algunas de las cuales se han conservado.

- Dichas traducciones romance de los Fueros de Aragón, por tanto, no son oficiales, aunque se hiciese mucho uso de ellas a lo largo de la Baja Edad Media; y los aragoneses, en cambio, por temor a sufrir mengua en sus derechos con errores en traducciones, mantuvieron siempre los Fueros de 1247 en su lengua latina original, tanto en las compilaciones oficiales manuscritas de los mismos, como en todas las ediciones impresas efectuadas entre los siglos XV y XIX.

- Se podrá reconstruir unas cuatro quintas partes de la versión última del Fuero Latino Extenso de Jaca, por comparación de los Fueros de Aragón de 1247 con la versión romance del Fuero Extenso de Jaca, única llegada completa a nuestros días.

- Situará definitivamente a la versión A del que a partir de ahora deberíamos denominar como Fuero Romance Extenso de Jaca, de comienzos del siglo XIV, en el lugar que verdaderamente le corresponde en este proceso evolutivo del texto, es decir, como una traducción de la última versión del Fuero Latino extenso de Jaca, efectuada con bastante posterioridad a la redacción de los Fueros de Aragón de 1247 e independientemente de éstos.

6.- En definitiva, la reconstrucción parcial de la versión primitiva latina del Fuero Extenso de Jaca, constituye una excusa perfecta para volver al principio 
y replantear de nuevo todo el tema de los orígenes del derecho foral aragonés; pero al mismo tiempo supone un fuerte impulso para seguir avanzando con mejor criterio y conseguir desmontar tópicos y errores que enmascaran el desarrollo de un proceso que todavía puede recuperarse en su práctica totalidad, haciendo una correcta interpretación de las abundantes fuentes documentales llegadas a nuestros días.

\section{Referencias bibliográficas}

Barrero García, Ana María (2003), «La difusión del Fuero de Jaca en el Camino de Santiago». En El Fuero de Jaca. Vol.II: Estudios. Zaragoza, El Justicia de Aragón: 113-160.

GARCía Edo, Vicente (2015), «Concordancias entre el Fuero de Estella de 1164 y los Fueros de Aragón de 1247», Glossae: European Journal of Legal History, 12: 359-380.

García Edo, Vicente (2016), «El Fuero de Zaragoza en el siglo XII. (Aproximación a su estudio)», Revista de Dret Històric Català, 15: 165-197.

LACARRA DE Miguel, José María (1982), Documentos para el estudio de la reconquista y repoblación del Valle del Ebro. Vol. 1. Zaragoza, Anúbar. Colección Textos Medievales, 62.

Lalinde Abadía, Jesús (1976), Los Fueros de Aragón. Zaragoza, Librería General.

Ledesma Rubio, María Luisa (1991), Cartas de población del reino de Aragón en los siglos medievales. Zaragoza, Institución Fernando el Católico.

Martínez Doral, Luis Alfonso (1971) «Notas sobre un texto para el estudio del derecho aragonés en la Edad Media», Revista de Historia Jerónimo Zurita, 21-22 (1971): 247-252.

Molno, Mauricio (1959-60) «La difusión del Fuero de Jaca». Boletín de la Real Academia de Buenas Letras de Barcelona, 28: 21-88

Molno, Mauricio (1964), El Fuero de Jaca. Zaragoza, Consejo Superior de Investigaciones Científicas.

Morales Gómez, Juan José y Manuel José Pedraza Gracia (1986) Fueros de Borja y Zaragoza. Edición crítica e índices. Zaragoza, Anúbar. Colección Textos Medievales, 74 .

PÉrez Martín, Antonio (1999) Los fueros de Aragón: la compilación de Huesca. Edición crítica de sus versiones romances, Zaragoza, El Justicia de Aragón.

Pérez Martín, Antonio (2010), Los Fueros de Aragón de 1247. La Compilación de Huesca. Edición crítica del texto oficial latino. Zaragoza, El Justicia de Aragón.

Ramos Loscertales, José María (1925) «Textos para el estudio del derecho aragonés en la Edad Media. Recopilación de Fueros de Aragón», Anuario de Historia del Derecho Español, 2 (1925): 491-523. 
Ramos Loscertales, José María (1927), Fuero de Jaca (última redacción). Barcelona, Facultad de Derecho.

Ramos Loscertales, José María (1928) «Textos para el estudio del Derecho Aragonés en la Edad Media. 1. Recopilación de Fueros de Aragón», Anuario de Historia del Derecho Español, 5 (1928): 389-411.

Real Academia de la Historia (1852), Colección de fueros y cartas pueblas de España por la Real Academia de la Historia. Madrid, Impr. De la Real Academia de la Historia.

SÁnchez Casabón, Ana Isabel (1995), Alfonso II, rey de Aragón, conde de Barcelona y marqués de Provenza. Documentos 1162-1196). Tomo II. Institución Fernando el Católico. (CSIC). Zaragoza.

Ubieto Arteta, Antonio (1975), Jaca: documentos municipales: 971-1269. Valencia, Anúbar.

Ubieto Arteta, Antonio (1989), «Los precedentes de los Fueros de Aragón». En Vidal Mayor. Estudios. Huesca, Diputación Provincial/Instituto de Estudios Altoaragoneses: $30-33$.

Viruete ERdozaín, Roberto (2013), La colección diplomática del reinado de Ramiro I de Aragón (1035-1064). Zaragoza, Institución Fernando el Católico. 